\title{
A Literacia Probabilística na Perspectiva da Teoria Histórico-Cultural
}

\author{
TREJO, Adriane Buchwitz Del ${ }^{1}$
}

\section{RESUMO}

Considerando que o homem é um sujeito histórico e social, este estudo bibliográfico possui uma abordagem metodológica de natureza qualitativa e delineamento descritivo explicativo, que tem como objetivo de relacionar os pressupostos da Teoria Histórico-Cultural (THC) com o ensino de Probabilidade nos anos iniciais do Ensino Fundamental focando no desenvolvimento da literacia probabilística e fazendo apontamentos correlacionando-os com referenciais curriculares. Assim, no decorrer do texto, tem-se a intenção do diálogo entre conceitos e ideias da Teoria Histórico-Cultural com os elementos cognitivos relacionados à literacia probabilística, apontando considerações dos referenciais curriculares do estado de São Paulo e nacionais. Como principais resultados, temos que os pressupostos desta teoria podem contribuir para uma prática educativa que priorize o aspecto social e para a aquisição de um conhecimento mais rico, mais ativo e mais significativo ao aluno.

\section{Teoria Histórico-Cultural. Ensino de Probabilidade. Literacia Probabilística. Anos Iniciais. Ensino Fundamental. \\ Probabilistic Literacy in the Historical-Cultural Theory Perspective}

\begin{abstract}
Considering that man is a historical and social subject, this bibliographic study has a qualitative methodological approach and explanatory descriptive design, which aims to relate the assumptions of Historical-Cultural Theory (THC) with the teaching of Probability in the early years of Elementary Education focusing on the development of probabilistic literacy and making notes correlating them with curricular references. Thus, throughout the text, the intention is to dialogue between concepts and ideas of Historical-Cultural Theory with the cognitive elements related to probabilistic literacy, pointing out considerations of the curricular frameworks of the state of São Paulo and nationally. As main results, we have that the assumptions of this theory can contribute to an educational practice that prioritizes the social aspect and to the acquisition of a richer, more active and more meaningful knowledge to the student.
\end{abstract}

\footnotetext{
${ }^{1}$ Mestranda em Educação pela Universidade Estadual Paulista Júlio de Mesquita Filho (UNESP). Professora I na Rede Municipal de Ensino de Presidente Prudente. E-mail: adriane.buchwitz@unesp.br. Lattes: http://lattes.cnpq.br/1850168197236399. Orcid: http://orcid.org/0000-0003-3284-194X.
}

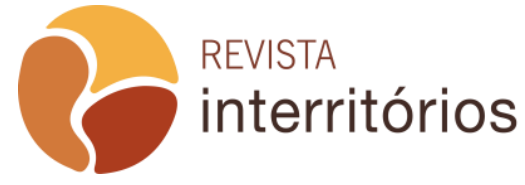

Interritórios | Revista de Educação Universidade Federal de Pernambuco, Caruaru, BRASIL | V.6 N.11 [2020] 
Historical-Cultural Theory. Probability Teaching. Probabilistic Literacy. Early

Years. Elementary School.

\section{La Literacia Probabilística en la Perspectiva de la Teoría Histórico-Cultural}

\section{RESUMEN}

Considerando que el hombre es un sujeto histórico y social, este estudio bibliográfico tiene un enfoque metodológico de naturaleza cualitativa y diseño descriptivo explicativo, cuyo objetivo es relacionar los presupuestos de la teoría histórico-cultural (THC) con la enseñanza de la probabilidad en los primeros años de la escuela primaria centrados en el desarrollo de la literacia probabilística tomando notas y correlacionándolas con referencias curriculares. Por lo tanto, a lo largo del texto, la intención es dialogar entre conceptos e ideas de la teoría histórico-cultural con los elementos cognitivos relacionados con la literacia probabilística, señalando consideraciones de las referencias curriculares del estado de São Paulo y nacionales. Como resultados principales, tenemos que los presupuestos de esta teoría pueden contribuir con una práctica educativa que prioriza el aspecto social y la adquisición de un conocimiento más rico, más activo y más significativo para el estudiante.

Teoría Histórico-Cultural. Enseñanza de Probabilidad. Literacia Probabilística. Años Iniciales. Enseñanza Fundamental.

\section{La Literacia probabilistica nella prospettiva della teoria storico-culturale}

\section{RIASSUNTO}

Considerando che l'uomo è un soggetto storico e sociale, questo studio bibliografico ha un approccio metodologico qualitativo e un disegno descrittivo esplicativo, che mira a mettere in relazione le ipotesi della teoria storico-culturale (THC) con l'insegnamento della Probabilità nel primi anni di scuola elementare concentrandosi sullo sviluppola literacia probabilistica e prendendo appunti correlandoli con i riferimenti al curriculum. Pertanto, in tutto il testo, l'intenzione è quella di dialogare tra concetti e idee della teoria storico-culturale con gli elementi cognitivi legati alla literacia probabilistica, sottolineando considerazioni sui riferimenti al curriculum dello stato di San Paolo e nazionali. Come risultati principali, abbiamo che i presupposti di questa teoria possono contribuire a una pratica educativa che privilegia l'aspetto sociale e all'acquisizione di una conoscenza più ricca, più attiva e più significativa per lo studente.

Teoria storico-culturale. Insegnamento della probabilità. Literacia probabilistica. Nei primi anni. Scuola elementare.

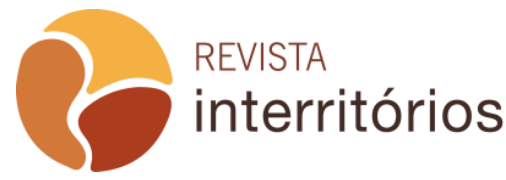




\section{INTRODUÇÃO}

Este presente artigo apresenta um estudo bibliográfico de natureza qualitativa, tem com o objetivo de relacionar os pressupostos da Teoria HistóricoCultural (THC) com o ensino de Probabilidade nos anos iniciais do Ensino Fundamental focando no desenvolvimento da literacia probabilística e fazendo apontamentos correlacionando-os com referenciais a nível estadual paulista (Currículo Paulista) e a nível nacional (Base Nacional Comum Curricular).

Ao longo do texto são discutidos ideias e conceitos embasados por Vygotsky (1896-1934) e os colaboradores da Teoria Histórico-Cultural, tais como Leontiev (1903-1979), Luria (1902-1977) e Davydov (1930-1998), entre outros autores que abordam dos mesmos pressupostos teóricos. Com isso destacamse as contribuições desses pesquisadores como referenciais teóricos para discutirmos a literacia probabilística na perspectiva da THC. A nível de esclarecimento, neste artigo adotaremos as grafias de Vygotsky e Davydov, salvo as citações e referências que abordaremos o que é proposto nelas.

A Teoria Histórico-Cultural baseia-se no materialismo histórico dialético difundido por Marx (1818-1883) e Engels (1820-1895). A THC surge nas primeiras décadas do século XX com o interesse de explicar o desenvolvimento humano, através da análise das funções psicológicas superiores (MARTINS, 2016, p. 44), considerando o homem como sujeito histórico e social.

Em relação ao nosso objeto de estudo, com a inserção do bloco temático de Tratamento da Informação, nos Parâmetros Curriculares Nacionais (PCN) em 1997 houve avanços à educação nacional no sentido de trabalhar com os conteúdos de probabilidade, estatística e combinatória desde as séries iniciais do Ensino Fundamental. Isso proporcionou o desenvolvimento de competências e habilidades desde o início de sua escolarização.

Em 2017, com a homologação da Base Nacional Comum Curricular (BNCC) definiu-se a denominação de unidade temática de Probabilidade e Estatística para os conteúdos do bloco temático de Tratamento da Informação.

Segundo a BNCC, esta unidade temática

[...] propõe a abordagem de conceitos, fatos e procedimentos presentes em muitas situações-problema da vida cotidiana, das ciências e da tecnologia. Assim, todos os cidadãos precisam desenvolver habilidades para coletar, organizar, representar, interpretar e analisar dados em uma variedade de contextos, de maneira a fazer julgamentos bem fundamentados e tomar as 
decisões adequadas. Isso inclui raciocinar e utilizar conceitos, representações e índices estatísticos para descrever, explicar e predizer fenômenos. (BRASIL, 2017, p. 272).

De forma semelhante à BNCC, o Currículo Paulista agrupa os conteúdos de estatística, probabilidade e combinatória na unidade temática de Probabilidade e Estatística que expõe que o ensino de Probabilidade

[...] envolve resolução de problemas de contagem e compreensão do princípio multiplicativo, o que favorece os estudantes a lidarem com situações que envolvam diferentes tipos de agrupamentos; favorece também o desenvolvimento do raciocínio combinatório e, assim, a compreensão de que muitos dos acontecimentos do cotidiano são de natureza aleatória. As noções de acaso e incerteza que se manifestam intuitivamente podem ser exploradas em situações em que os estudantes realizam experimentos e observam eventos. (SÃO PAULO, 2019, p. 326).

Por se tratar de uma unidade temática que abrange três conteúdos ao longo de todo o processo de escolarização dos alunos, e com a intenção de fazer um recorte na amplitude da unidade temática de Probabilidade e Estatística, abordaremos nesse artigo o ensino de Probabilidade nos anos iniciais do Ensino Fundamental, na perspectiva do desenvolvimento da literacia probabilística.

Diante ao exposto, esta pesquisa destacará conceitos e ideias de autores dentro da perspectiva da THC, bem como associados ao ensino de Probabilidade e aos referenciais curriculares, e, portanto, visando constituir um embasamento teórico para a aquisição da literacia probabilística nos anos iniciais do Ensino Fundamental.

A seguir, descreveremos as ações metodológicas abordadas neste estudo.

\section{Metodologia}

Com a intenção de apresentar e discutir as contribuições da THC para o desenvolvimento da literacia probabilística, este artigo é pautado pela abordagem metodológica de natureza qualitativa (CRESWELL, 2010) com delineamento descritivo explicativo (GIL, 2008).

Em relação aos procedimentos metodológicos, este artigo adotou enquanto instrumentos de coleta de dados a revisão de literatura.

Segundo Creswell (2010) a revisão de literatura auxilia os estudos de um determinado tópico, pois limita o escopo da sua investigação e transmite aos leitores a importância do seu estudo. O autor também evidencia que um dos

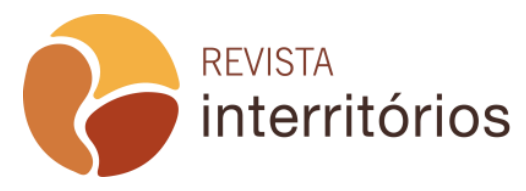

Interritórios | Revista de Educação Universidade Federal de Pernambuco, Caruaru, BRASIL | V.6 N.11 [2020] 
objetivos da revisão de literatura é compartilhar os resultados de estudos anteriores que estão relacionados ao estudo que está sendo relatado.

Desta forma a presente revisão de literatura foi desenvolvida da seguinte forma. Como tema, o nosso objeto de estudo definiu-se como a literacia probabilística na perspectiva da THC. O nosso levantamento bibliográfico levou em conta a pesquisa bibliográfica de estudos, de pesquisas e referenciais curriculares pertinentes ao tema. A seguir, realizamos a leitura pormenorizada identificando as principais ideias e descrevendo-as de forma sintética. Ao analisarmos e interpretarmos os dados encontrados, seguimos com as descrições e as considerações que serão expostas ao longo do texto, enquanto descrições analíticas.

Desse modo, as descrições analíticas foram elaboradas pela articulação das informações coletadas com reflexões, de acordo com o referencial teórico e em pesquisas relativas ao tema.

A partir dessas considerações, seguiremos com a apresentação de referenciais teóricos que embasaram este estudo e das descrições analíticas elaboradas.

\section{A Teoria Histórico-Cultural e a Literacia Probabilística}

O processo de ensino de Probabilidade nos anos iniciais do Ensino Fundamental, assim como os demais períodos escolares, tem como intuito promover uma compreensão de que nem todos os fenômenos são determinísticos, centrando o trabalho no desenvolvimento da aleatoriedade (BRASIL, 2017, p. 272).

Diante a essa premissa, a BNCC traz em sua redação os conteúdos de Probabilidade desde o $1^{\circ}$ ano do Ensino Fundamental, noções que vão sendo retomadas, ampliadas e aprofundadas, ano após ano (BRASIL, 2017, p. 278279), elencando-os com as situações cotidianas, mostrando que nem todos os fenômenos são determinísticos. O pensamento exposto neste documento curricular oficial contrapõe-se a uma visão positivista, uma educação tradicionalista, a qual a Teoria Histórico-Cultural (THC) pretende diluir com rigidez.

Na THC o homem é entendido como sujeito histórico. Para Vygotky (1991) esse sujeito histórico não é apenas ativo, mas também interativo, porque os seus conhecimentos são constituídos e organizados a partir de relações com os objetos e intra e interpessoais. Corroborando com essa afirmação, Leontiev (1978, p. 261) ressalta que "o homem é um ser de natureza social, que tudo o que tem de humano nele provém da sua vida em sociedade, no seio da cultura criadas pela humanidade". As ideias de Vygotsky e Leontiev condizem com o 
pensamento de que é preciso elencar o ensino de Probabilidade, assim como outras áreas, como as situações do cotidiano.

Motivados pela constatação de que o ensino de Probabilidade deve ser abordado desde os anos iniciais do Ensino Fundamental e em relação a importância e uso desses conteúdos na vida social, de acordo com os documentos curriculares, prosseguiremos com as exposições acerca das contribuições da THC para essa abordagem temática.

Os conteúdos desenvolvidos durante os anos iniciais do Ensino Fundamental, na disciplina de Matemática, visam contribuir para 0 desenvolvimento cognitivo do aluno, oferecendo competências e habilidades, no sentido de que ele prossiga com seus estudos e com a intenção de ser alfabetizado e letrado matematicamente.

Em um entendimento de ir além de alfabetizar e letrar, com as competências e usos da leitura e da escrita associadas com suas funções na formação e acumulação de procedimentos, temos o conceito de literacia (OSLON, 1984 apud ODY; VIALI; 2013, p. 2013).

Voltamo-nos ao nosso objeto de estudo, mostra-se necessário o desenvolvimento da literacia probabilística com o indivíduo. Nessa perspectiva, Gal (2005) propõe um modelo composto por cinco elementos cognitivos que pretendem propiciar esse desenvolvimento.

O primeiro elemento - grandes tópicos - refere-se à abordagem de conteúdos tais como previsão, variação, incerteza, conceitos esses que permitem ao aluno a representar e interpretar o seu cotidiano através de informações probabilísticas (GAL, 2005).

Nesse sentido, na THC sobre conceitos, temos a contribuição de Davydov (1982) que propõe que o ensino consiste no desenvolvimento dos conceitos teóricos, em detrimento ao pensamento empírico, de uma forma em que os conceitos cotidianos e os conceitos científicos inter-relacionem-se.

Prosseguindo com a nossa descrição, temos o elemento cognitivo cálculos probabilísticos em que Gal (2005) considera a compreensão do sujeito diante às maneiras de calcular a Probabilidade dos eventos para o entendimento das informações transmitidas por outras pessoas e que possam através desses conhecimentos se comunicarem.

Tomando-nos novamente pelas ideias de Davydov, que considera que o ensino deve contemplar a formação de conceitos, temos que

O pensamento teórico tem seus tipos específicos de generalização e abstração, seus procedimentos de formação dos conceitos e operações com eles. Justamente, a formação de tais conceitos abre aos escolares o caminho para dominar os 
fundamentos da cultura teórica atual. [...] A escola, a nosso juízo, deve ensinar às crianças a pensar teoricamente. (DAVYDOV, 2002, p. 51-60).

Vygotsky (1987) afirma que o fato de uma criança explicar com convencimento um determinado conceito, questões ou resultados, deve-se principalmente pela ação do professor, pois o que se aprende na escola é, ou deveria ser hierarquicamente sistematizado.

Relacionado com a comunicação, afirmada anteriormente no elemento de cálculos probabilísticos, adentramos ao terceiro elemento proposto por Gal (2005) que é a linguagem probabilística. Segundo o autor, esse domínio é importante, pois os alunos precisam representar e comunicar, de forma a serem compreendidos os resultados probabilísticos.

Conforme Leontiev (1978, p. 267),

As gerações humanas morrem e sucedem-se, mas aquilo que criaram passa às gerações seguintes que multiplicam e aperfeiçoam pelo trabalho e pela luta as riquezas que lhes foram transmitidas e 'passam pelo testemunho' do desenvolvimento da humanidade.

Para que essa transmissão ocorra de fato, é imprescindível a linguagem que é "[...] um complexo sistema de códigos que designa objetos, características, ações ou relações; códigos que possuem a função de codificar e transmitir a informação introduzi-la em determinados sistemas [...]" (LURIA, 1986, p. 25). Dessa forma, no ambiente escolar, a linguagem deve assumir uma função social em que transmita o conhecimento de uma forma compreensível aos outros.

Acerca do elemento contexto, para o desenvolvimento da literacia probabilística, está relacionado com a noção de conhecimento do mundo, em outras palavras, pela compreensão de acaso e probabilidade que ocorre em situações cotidianas (GAL, 2005).

Conforme Leontiev (1978)

Cada geração começa, portanto, a sua vida num mundo de objetos e de fenômenos criados pelas gerações precedentes. Ela apropria-se das riquezas deste mundo participando no trabalho, na produção e nas diversas formas de atividade social e desenvolvendo assim as aptidões especificamente humanas que se cristalizaram, encarnaram neste mundo. (LEONTIEV, 1978, p. 265).

Dessa forma, mostra-se evidente que anteriormente à escola, o aluno possui um conjunto de conhecimentos culturalmente construídos e vividos e cabe a essa instituição sistematizá-los. 
Corroborando com a ideia de Gal (2005) sobre o elemento contexto, temos que

[...] se pergunta de onde nascem, como se formam, de que modo se desenvolvem os processos superiores do pensamento infantil, devemos responder que surgem no processo de desenvolvimento social da criança, por meio da translação a si mesma de formas de colaboração que a criança assimila durante a interação com o meio social que a rodeia. (VYGOTSKI, 1997, p. 217 apud MOURA et al., 2010).

Por fim, o último elemento cognitivo para o desenvolvimento da literacia probabilística é o referente às questões críticas que envolvem os questionamentos e a validação das informações probabilísticas (GAL, 2005).

Traçando um paralelo com a THC, a atividade consciente do homem é movida por motivos sociais. Com base nesse aspecto, Luria aponta que

Sabe-se que o homem pode refletir as condições do meio de modo imediatamente mais profundo do que o animal. Ele pode abstrair a impressão imediata, penetrar, nas conexões e dependências profundas das coisas, conhecer a dependência causal dos acontecimentos e, após interpretá-los, tomar como orientação não impressões exteriores, porém mais profundas. (LURIA, 1979, p.72).

Deste modo, para desenvolver-se a criticidade e a validação dos conhecimentos probabilísticos, precisa-se da inserção neste meio e um conhecimento mais aprofundado.

Gal (2005) remete a ideia de para que se vislumbre a literacia probabilística no Ensino de Probabilidade, os cinco elementos expostos devem se interagir mutuamente, e que as experiências de aprendizagem e avaliação escolar precisam ser significativas aos alunos e assim "exige-se atenção para a questão da transferência de competências na sala de aula para aprender situações fora da sala de aula" (GAL, 2005, p. 58).

Com isso, a finalidade de estudo de Probabilidade exposta pela BNCC (2017) é condizente aos elementos cognitivos apresentados por Gal (2005) e os pressupostos da THC em diálogo neste artigo. Para o documento curricular,

No que concerne ao estudo de noções de probabilidade, a finalidade, no Ensino Fundamental - Anos Iniciais, é promover a compreensão de que nem todos os fenômenos são determinísticos. Para isso, o início da proposta de trabalho com probabilidade está centrado no desenvolvimento da noção de aleatoriedade, de modo que os alunos compreendam que há eventos certos, eventos impossíveis e eventos prováveis. É muito comum que pessoas julguem impossíveis eventos que 
nunca viram acontecer. Nessa fase, é importante que os alunos verbalizem, em eventos que envolvem o acaso, os resultados que poderiam ter acontecido em oposição ao que realmente aconteceu, iniciando a construção do espaço amostral. (BRASIL, 2017, p. 272).

Portanto, mostra-se relevante 0 ensino de Probabilidade numa perspectiva da $\mathrm{THC}$ em que se pretenda alcançar o desenvolvimento da literacia probabilística nos alunos dos anos iniciais do Ensino Fundamental.

\section{Considerações Finais}

Este artigo teve por finalidade apresentar e discutir as contribuições da Teoria Histórico-Cultural (THC) para a literacia probabilística no ensino de Probabilidade nos anos iniciais do Ensino Fundamental.

A partir das leituras e da análise dos estudos levantados sobre a THC, sobre a literacia probabilística e os referenciais curriculares, foram evidenciados alguns dados pertinentes ao nosso estudo, que serão expostos a seguir.

Desse modo, quando o processo de ensino e aprendizagem é apoiado na THC, mostra-se que o conhecimento derivante é mais ativo, mais rico e mais significativo facilitando a relação do homem com o mundo, conduzindo a uma melhor assimilação do conteúdo.

Com esse diálogo permitiu-se também reconhecer que as contribuições da THC para o ensino de probabilidade nos anos iniciais possibilitam uma reorientação didática e pedagógica com vistas para se alcançar a literacia probabilística. Logo, esses fatos encontram-se de acordo com a ideia defendida por Davydov (1982) que o ensino na escola prioriza o caráter empírico e a generalização dos conhecimentos é dada na observação das características comuns dos fenômenos ou objetos, transformando os conceitos cada vez mais abstratos e mais distantes da sua natureza, o que nos faz refletir sobre a necessidade de se rever práticas e posturas enquanto educadores.

Na busca de dialogar a THC com a literacia probabilística, evidenciou que é possível o desenvolvimento da criança através de conceitos produzidos historicamente, contribuindo assim para uma aprendizagem pelos alunos de Probabilidade desde os primeiros anos de escolarização.

Através do diálogo textual presente neste artigo, entre a THC e a literacia probabilística, notamos que os pressupostos dessa teoria, podem contribuir para uma prática educativa que priorize o aspecto social, superando o caráter biológico preconizado por muito tempo na questão do desenvolvimento das funções psíquicas superiores e em relação ao caráter abstrato do ensino das ciências exatas.

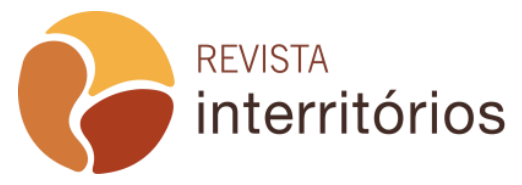




\section{REFERÊNCIAS}

BRASIL. Secretaria de Educação Fundamental. Parâmetros Curriculares Nacionais: matemática. Brasília: MEC/SEF, 1997.

BRASIL. Secretaria de Educação Básica. Base Nacional Comum Curricular: educação é a base. Brasília: MEC, 2017.

CRESWELL, J. W. Projeto de Pesquisa: métodos qualitativo, quantitativo e misto. Porto Alegre: Artmed, 2010.

DAVYDOV, V. V. Tipos de generalizacion en la enseñanza. Havana: Pueblo y Educacion, 1982.

DAVYDOV, V. V. El aporte de Leontiev al desarrollo de la psicología. In: GOLDER, M. (Org.). Angustia por la Utopía. Buenos Aires: Ateneo Vigotskiano de la Argentina, 2002. p. 51-60.

GAL, I. Towards Probability Literacy For All Citizens: building blocks and instructional dilemas. In: JONES, G., (ed.). Exploring Probability in Schools: challenges for teaching and learning. Springer, New York, p. 39-63, 2005.

GIL, A. C. Métodos e Técnicas de Pesquisa Social. 6. ed. São Paulo: Atlas, 2008.

LEONTIEV, A. O Desenvolvimento do Psiquismo. Lisboa: Horizonte, 1978.

LURIA, A.R. A Atividade Consciente do Homem e Suas Raízes Histórico-sociais. In: LURIA, A.R. Curso de Psicologia Geral - vol 1. Rio de Janeiro: Civilização Brasileira, 1979, p.71-84.

LURIA, A. R. Pensamento e linguagem: as últimas conferências de Luria. Trad. Diana Myriam Lichtenstein e Mario Corso. Porto Alegre: Artes Médicas, 1986.

MARTINS, L. M. Fundamentos da Psicologia Histórico-Cultural e da Pedagogia Histórico-Crítica, 2012. Disponível em:

http://ead.bauru.sp.gov.br/efront/www/content/lessons/78/Fundamentos\%20da\%20PH C\%20e\%20da\%20PHC\%20-\%20MARTINS.pdf Acesso em: 05 out. 2019.

MOURA, M. O. et al. A atividade orientadora de ensino: unidade entre ensino e aprendizagem. Revista Diálogo Educacional, Curitiba, v. 10, p. 205-229, jan./abr. 2010.

ODY, M. C; VIALI, L. Alfabetização, Letramento e Literacia: da aquisição e das habilidades de leitura, de escrita e cálculo, à utilização de suas competências na estatística e na probabilidade. In: CONGRESSO IBERO AMERICANO DE EDUCAÇÃO MATEMÁTICA, 7, 2013, Actas [...], Montevidéu, Uruguai, 2013.

SÃO PAULO (Estado). Secretaria de Estado da Educação. Currículo Paulista. São Paulo: SEDUC-SP, 2019.

VYGOTSKY, L. S. Pensamento e Linguagem. São Paulo: Martins Fontes, 1987.

VYGOTSKY, L. S. A Formação Social da Mente. São Paulo: Martins Fontes, 1991. 